\title{
Duby: Dual Binding Semantics
}

\author{
YOORI RAPOPORT \\ yoori.rapoport@gmail.com
}

December 8, 2020

\begin{abstract}
Reference counting is a simple and efficient method for garbage collection. One disadvantage of reference counting is the possible creation of reference cycles. We propose a dynamically typed compiled programming language called Duby, in which values can be bound to either an automatic variable or to a unique hierarchical path. The separation of variable binding forms allows Duby to implement reference counting for automatic variables which refer to values bound to unique hierarchical paths without creating reference cycles. Another advantage of explicit binding of values to unique hierarchical paths is the ability to provide a paradigmatic implementation of programming concepts using filesystem-like abstractions.
\end{abstract}

\section{INTRODUCTION}

Values in programming languages are bound to variables [1]. Languages that support dereferencing (i.e. pointers) allow binding variables to an address of a value rather than to the value itself [18].

The variables are defined in scopes: scopes represent code blocks in which the variables exist. Automatic variables are variables that release the values bound to them from memory upon the destruction of their scope.

It is easier to manage memory when all variables are automatic since the timing of destruction of every value is known in advance. However, some values are required to persist beyond the lifetime of the scope where they were created.

In such cases, automatic variables cannot be used to store those values and instead their lifetime is either controlled manually by the programmer or automatically by a memory management mechanism embodied in the programming language.

Both solutions have their disadvantages: automatic memory management adds complexity to the language runtime and manual memory management complicates the programming process.
Manual memory management is error-prone due in part to the fact that memory addresses are big integer numbers, which are assigned during program execution.

We propose a programming language which provides two ways to bind values: they can be either be bound to automatic variables or to hierarchical paths.

The language is called Duby; its syntax and semantics in aspects unrelated to variable binding and memory management are based on Python [28].

However, all values in Duby can be separated by the binding method used upon their creation into three categories: unbound, bound to an automatic variable, and bound to a hierarchical path. This separation allows Duby to implement a cycle-free reference counting of values in the latter category.

Explicit binding of values to unique hierarchical paths is the ability to provide a paradigmatic implementation of programming concepts using filesystem-like object representations, which could be beneficial in programming education. 


\section{i. Overview}

First we introduce Duby's syntax and semantics and provide basic usage examples of the language.

In the language implementation and benchmarks section we describe the tools we use to implement Duby and provide some basic language benchmarks.

Then we describe the previous related work and, finally, propose directions for future development.

\section{SYNTAX}

Duby's syntax is based on Python. Every syntactically valid Python construct is syntactically valid in Duby as well. Python language is described in official Python documentation [28]; the full grammar specification is available online ${ }^{1}$

Beside Python code, Duby code may also contain branch declarations.

Branch declaration start with a / character.

The first line of a branch declaration must be followed by an indented block of a YAML graph declaration, defined according to YAML specification [6], with the following restrictions: explicit YAML tagging of node types is not allowed and node anchors and references are not allowed. Therefore, YAML graph in a branch declaration defines a tree.

YAML nodes can be of any of the following types defined by the YAML failsafe and core schemas ${ }^{2}$ Mapping, Sequence, Null, Boolean, Integer, Float (i.e. Floating Point type).

YAML strings are further tokenized using the following rules: a String is a YAML string which begins and ends with either " or ' and a PathSeq is any other string. PathSeq $\mathrm{s}$ are further split into a sequence of Path strings, where a Path string is either a string which does not contain white spaces or

\footnotetext{
${ }^{1}$ https://docs.python.org/3/reference/grammar.html

${ }^{2}$ https://yaml.org/spec/1.2/spec.html\#Schema
}

which begins and ends with a back quote ( ') sign.

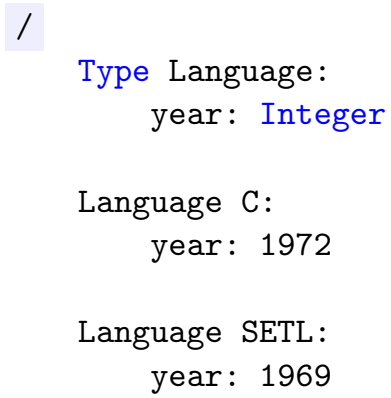

The branch declared above is depicted in Figure 1 The figure describes node names, keys, paths and declared types, which are discussed later on, as well.

\section{i. Node Path Definition}

For every branch declaration the value of the root node in YAML tree graph must be of type Mapping .

Every child node of a Mapping has a key and a value. Every node key must be of the type PathSeq and we call the last path in that sequence the node name.

For every item in a Sequence node, its node name is its index in the sequence (starting with $0)$.

Node names cannot contain / or : characters.

Every compiled module in Duby has a unique identifier string which is called the local prefix. We will denote the local prefix by / .

Additionally, the identifier of a module which contains all built-in and system-wide definitions is called the global prefix. We will denote the global prefix by // .

The path of a child node of a root in a branch declaration is the local prefix followed by the node name. The path of a node that is not a child of a root in a branch declaration is the path of its parent node followed by / followed by the node name.

Every node in every branch declaration must have a unique path string: declaring two nodes with the same path string is illegal. 


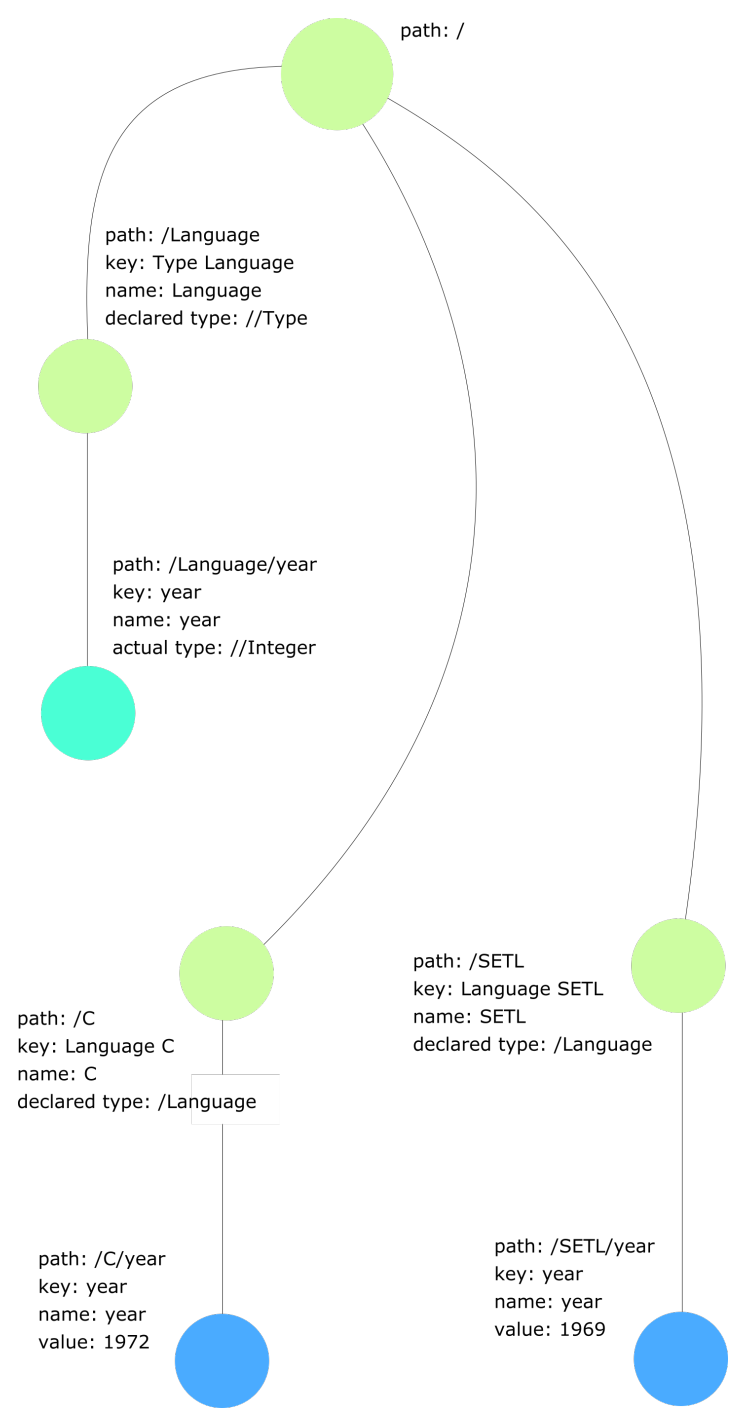

Figure 1: Branch Declaration Nodes

Legend: symbolizes a Mapping node, symbolizes an Integer node and symbolizes a String node.
For instance, the path /Adam/loves in the branch declared in Figure 2 identifies a node whose name is loves and whose value is the path / Jane .

After node names are determined, every PathSeq can be converted to single path string by applying the following rules on the first value in the sequence and then concatenating it with rest of the sequence: if the value starts with a single $/$, then its first character is removed and then it is prefixed by the local prefix; if the value starts with ./ or // then it describes a filesystem path of another module and is replaced by the local prefix of that module; if prefixing the value by the local prefix identifies a node then it is replaced by the path of that node; otherwise, if prefixing the value by the global prefix identifies a node then it is replaced by the path of that node; if none of the previous rules can be applied on a PathSeq then it is illegal.

For instance, in Figure 2, the path IAO, which is used inside the branch node /Adam/personality, is replaced by the path /IAO .

\section{ii. Modifiers}

Branch nodes of a non-aggregate type (neither a Mapping nor a Sequence) are called scalar nodes.

Values of scalar nodes in a branch declaration may optionally end with a modifier. Allowed modifiers are: [child] and [path] . Modifiers are not considered to be part of node values; they are parsed before node values are evaluated.

In the sample branch declaration described in Figure 2, the path /Person/loves has a modifier [path].

\section{iii. Sharing Branch Declarations}

Branch declarations can be shared. For instance, suppose that the following code is saved under the file name decl.by: 


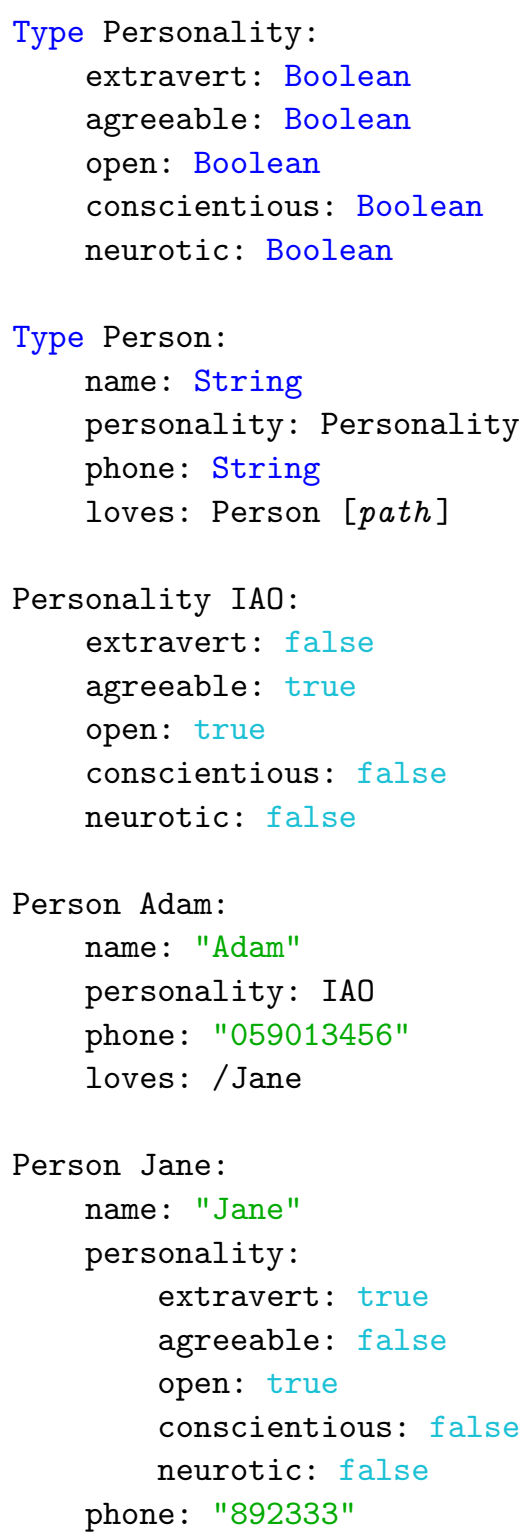

Figure 2: Branch Declaration

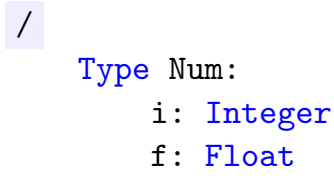

then it could be used in the following way from any other file in the same directory:

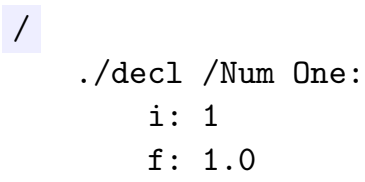

\section{TYPES}

\section{i. Node Types}

If the key of a node of type Mapping in a branch declaration contains two path strings or more, then the first path string is its declared type. A node which has a declared type is called a typed node.

A typed node with a declared type //Type is called a type declaration.

The branch declaration in Figure 2 has two type declarations: /Personality and /Person. The declared type of the nodes /Adam and Jane is /Person. The declared type of the node /IAO is /Personality.

The declared type of any typed node must be a path of a type declaration or //Type .

The actual type defined by a child node of a type declaration is deduced using following rules from the value and the modifier of the node:

- If there is no modifier then the actual type is the value.

- If the modifier is [child] then the actual type is a reference to another (child) node of the type defined by the value.

- If the modifier is [path] then the actual type is a path of another node of the type defined by the value.

For instance, in Figure 2 we can see that the actual type of //Personality/extravert 
is //Boolean and the actual type of $/ /$ Person/loves is a //Path to another node of type /Person .

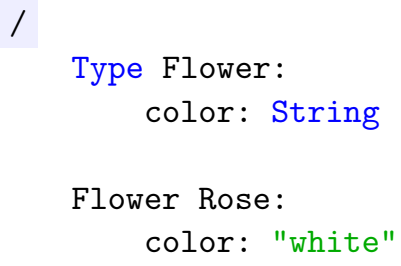

In the sample above, a type /Flower is declared and then a node of that type is also declared.

Using a value of type other than String as a color of nodes with a declared type Flower would produce an error.

\section{ii. Type System}

Type system in Duby is based on the C language types. The formal definition of $C$ language is provided in [22]. Every declared type has a corresponding $C$ structure.

However, Duby supports dynamic typing: every value in Duby is bundled with a reference to its type structure, which can be accessed using a built-in function type () . Every type structure contains the following functions: getattr(), which allows retrieving structure fields by their names at runtime, $\operatorname{copy}()$, which creates a copy of a value, and assign(), which replaces one value by another value of the same type. The functions hasattr( $)$ and setattr(), which check for existence of a field name and change the value of a field by its name in a structure, respectively, exist as well in every type structure. Further, additional functions, such as $\operatorname{delattr}()$, exist in every type structure, however, their default implementation always raises an error.

On top of $C$ types, Python-like classes as well as YAML types are built. Figure 3 lists built-in types in Duby and their C, YAML and Python (with ctypes module) counterparts.

For values of types object and Mapping, the function setattr( $)$ allows adding new field names and changing field types and the function delattr() removes access to a field with the given name at runtime.

\section{SEmantics}

The semantics of Duby is based on Python. However, there are certain divergences, which are a consequence of Duby's approach to memory and variable management.

\section{i. Automatic Variables}

All function variables in Duby are automatic: their lifetime is bound by the lifetime of the function scope.

In the following example, different two different numbers will be printed for $\mathrm{u}$ and for $\mathrm{v}$ since the id() function in CPython (the official reference implementation of Python) and in Duby returns the address of the value in memory (an unsigned integer number):

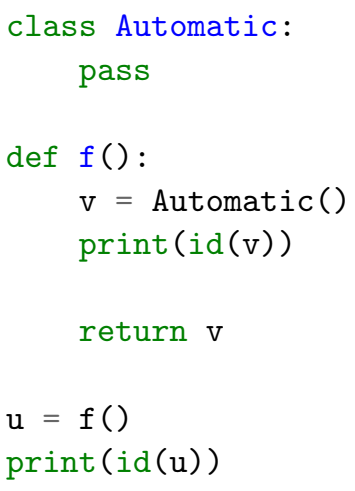

Instance attributes, dictionary keys and values, list items and set elements in Duby are automatic as well: their lifetime is bound by the lifetime of their scope.

\section{ii. Assignments}

When an automatic variable is assigned, a deepcopy of its value is performed and is actually assigned.

The code below assigns a new list to a variable $\mathrm{x}$. Then it assigns the variable $\mathrm{x}$ to a variable $\mathrm{y}$ and changes the first element of a 


\begin{tabular}{llll} 
Duby & C & YAML & Python \\
\hline int & long & Integer & ctypes.c_long \\
\hline float & double & Float & ctypes.c_double \\
\hline bool & bool & Boolean & ctypes.c_bool \\
\hline None & & Null & None \\
\hline object & & object \\
\hline list & & list \\
\hline dict & & dict \\
\hline str & & str \\
\hline class & String & class \\
\hline Sequence & & Sequence & \\
\hline Mapping & & Mapping & \\
\hline Type & & ctypes.Structure \\
\hline
\end{tabular}

Figure 3: Types in Duby, C, YAML and Python

list bound by the variable $\mathrm{x}$. Finally, it prints the first element of a list bound by the variable $\mathrm{y}$.

$$
\begin{aligned}
& x=[1,2,3,4,5,6,7] \\
& y=x \\
& x[0]=999 \\
& \operatorname{print}(y[0])
\end{aligned}
$$

When running the code above in Python interpreter, the output is 999 . However, the output of a compiled Duby program above is 1 .

The reason for the difference is that in Python the statement $\mathrm{y}=\mathrm{x}$ binds the variable $y$ to the same list bound by the variable $\mathrm{x}$.

However, in Duby the assignment statement copies the list bound by variable $\mathrm{x}$ into a new list and binds the new list to the variable $\mathrm{y}$. Since the first list is changed after the assignment, this change does not affect the new list.

\section{iii. Closures}

Closures in Duby are not allowed; functions and classes can be defined inside other functions. However, nested functions and classes cannot access the variables of the wrapping function.

The following code snippet does not compile in Duby, since the variable external belongs to the scope of $g()$, which is not visible in $f()$.

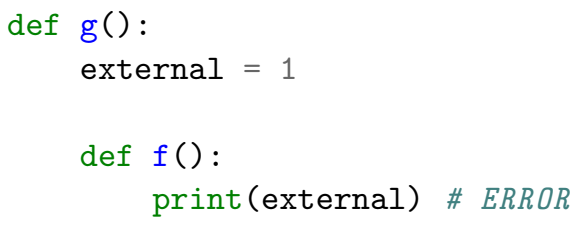

On the other hand, global variables are visible to all functions in a module.

Therefore, the following code snippet produces the same output in Duby and in Python. 


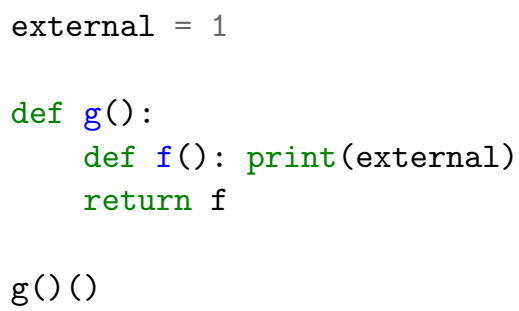

\section{iv. Generators}

When a generator is initialized in Duby deepcopies of all its arguments are created.

Therefore, the output of the following code snippet would be $[0,0,0]$ despite the fact that the list passed to the generator is modified afterwards.

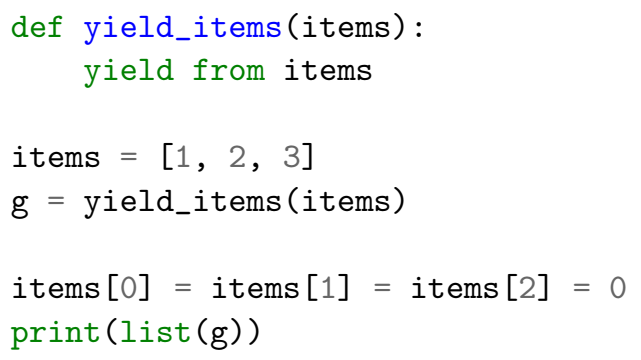

When using a comprehension syntax inside parenthesis, a tuple is created (and not a generator, as in Python). However, if implementing the special methods ___map_-_ () and _-_reduce _-_ () allows the object to be used in a comprehension:

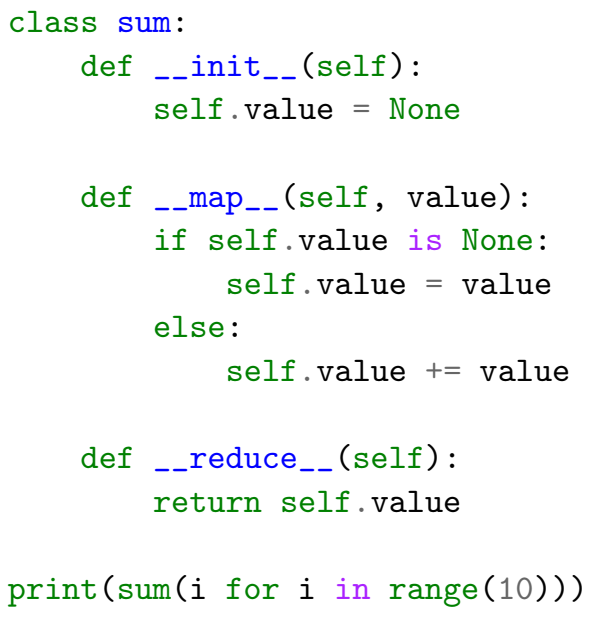

The value 45 is printed in the sample above.

\section{v. Memory Nodes}

Automatic variables in Duby cannot be dereferenced.

However, beside automatic variables, values can be bound to a memory tree path. A memory tree is a mapping of path strings to values.

One way to bind a value to a memory tree path is to use the branch declaration syntax.

When a branch declaration is read, the child nodes of its root are automatically bound to a memory tree path.

Branch declaration nodes are bound to memory tree paths recursively: a child of a typed node that has a child modifier in the corresponding type declaration, a child node of a Mapping, and a child node of a Sequence are bound to a memory tree path as well.

All branch declaration nodes, which are bound to a memory tree path, are bound to a path that is equal to their path in the branch declaration.

We say that a memory node is a value which is bound to a memory tree path.

When a child node of the root of a branch declaration is bound to a memory tree path, value of type Proxy, which implements a proxy pattern on the memory node, is bound to the scope of a module as well: variable name of the Proxy object equals the node name of the memory node it represents.

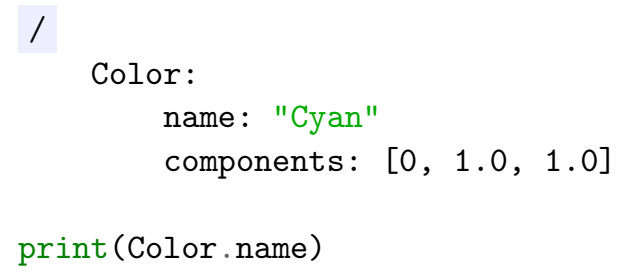

The output of the sample above would be:

$$
\text { Cyan }
$$

Values of type Proxy cannot be converted to memory nodes themselves: a Proxy value can only be bound to an automatic variable. 
The following code snippet dynamically modifies the node /Color declared in the previous sample.

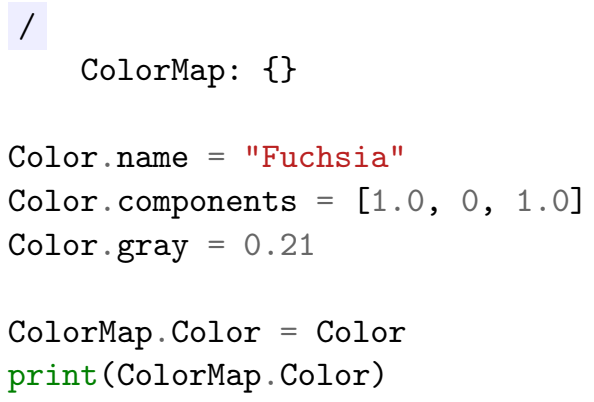

As seen in the example above, Proxy instances can be used to bind values to paths in the memory tree as well: the value 0.21 is bound to the path /Color/gray .

However, when assigning a Proxy value to an attribute of a Mapping or an item in a Sequence, then, unless the node it represents is an orphan, the path of that node is assigned instead.

Therefore, the output of the code above is /ColorMap/Color (the local prefix is actually printed instead of / ).

Path strings can be converted back to Proxy objects manually. Therefore, the previous sample could be modified further in the following way:

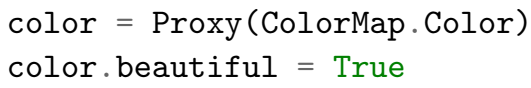

The local keyword can be used to get the local prefix string.

\section{Memory Management}

\section{i. Unbinding Memory Nodes}

Memory nodes can be explicitly unbound from the memory tree using the builtin unbind() function. If a memory node is unbound then all its child nodes are also unbound.

Memory nodes which are unbound from a memory tree are bound to a garbage collector.
A node is a value which is bound to either a memory tree or a garbage collector.

A node, which is bound to a garbage collector, and which is not a child of another node, is deallocated if no Proxy instances refer to it. When a node is deallocated its child nodes are orphaned and may also be deallocated in a recursive process.

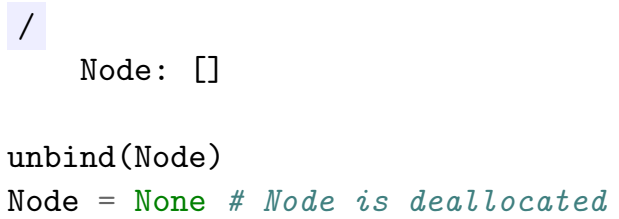

Reference counting is used to prevent destruction of nodes which are referenced by Proxy values. Creation or destruction of Proxy instances increases or decreases, respectively, the corresponding reference counter.

Therefore, the following code works and prints Beige.

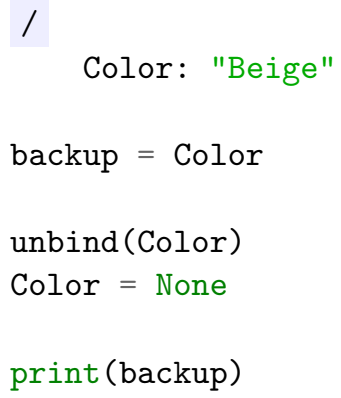

Lemma 1. Automatic built-in reference counting in Duby does not produce reference cycles.

Proof. By Duby's design, only nodes have reference counters. Moreover, node-to-node references always form a forest: only a parent node can reference its child nodes. The only other values that can have references to nodes are values of type Proxy. A Proxy value can be bound only to automatic variables and therefore it cannot be a node.

Let $H$ be the set of all nodes and let $P$ be the set of all values of type Proxy which exist in program memory at a specific point in time during program execution. 
Let $G=(P \cup H, E)$ be a digraph where $E$ is the set of pairs $(p, h) \in(P \cup H) \times H$ for which $p$ stores a reference to $h$.

Let $C$ be the set of all vertices $v \in P \cup H$ for which there is a cycle $S$ in $G$ such that $v$ lies on S.

Since node-to-node references always define a forest, there cannot be a cycle in $G$ which contains only vertices in $H$. Therefore, every cycle in $G$ must contain a vertex $p \in P$ and, therefore, if $P \cap C=\varnothing$ then there are no cycles in $G$.

$P \cap H=\varnothing$ since a Proxy value can never be a node.

Suppose that $p \in P$, which means that $p \notin H$ and, consequently, that for every vertex $v \in$ $P \cup H$ of $G$ there is no edge $(v, p) \in E \subseteq(P \cup$ $H) \times H$. Therefore, $p$ cannot lie on a cycle in G.

Thus $P \cap C=\varnothing$, which means that there are no cycles in $G$.

\section{ii. Rebinding Memory Nodes}

Nodes can be rebound to a different memory tree path: first they must be unbound from their original path and then bound to the new path, as is demonstrated in the following example:

The output of the sample above is yellow leaf .
}

\section{IMPLEMENTATION AND BENCHMARKS}

\section{i. Using $\mathrm{C}++$ as an Intermediate Lan- guage}

We use an experimental code generator written in Python, which generates $\mathrm{C}++17$ code from Duby programs. This code generator does not implement Python language features that are not required to demonstrate dual binding semantics. However, $\mathrm{C}++$ code for all samples and benchmarks in this paper was generated.

$C$ programming language has been often been used as an intermediate language for experimental language compilers [16, 25].

We use $\mathrm{C}++$ due to the following integrated features that are not available in the $C$ language: stack unwinding and exception handling, templates, constant expressions and lambda expressions [17].

Using $\mathrm{C}++$ as an intermediate language simplifies the integration with environments which provide their own $\mathrm{C}++$ compilers. If the vendor provides its own compiler then writing a frontend which is aimed at an intermediate language of another infrastructure may make the integration of code written in Duby with the libraries of that vendor harder. One example of such framework is Emscripten, which provides its own LLVM based emcc compiler [33].

\section{ii. Memory Pools}

Duby's runtime uses two alternating memory pools for allocation of values that are bound to automatic variables. Every pool has a doublelinked list of allocated chunks. List entry of every allocated chunk of the memory in a pool is added at the head of the corresponding list. The address of every consequent item in a list of a memory pool must be lower then the address of the previous item. If a size of a chunk in an allocation request is too big to be inserted at the head of one of the memory pools' lists, then system-wide malloc() function is used to allocate the chunk. 


\section{iii. Benchmarks}

We compared Duby's performance to CPython using the following algorithms:

- Fibonacci Sequence: calculating the $20^{\text {th }}$ element of a Fibonacci sequence for $10^{3}$ times.

- Quicksort: sorting an array of 5000 uniform random floating point numbers for $10^{3}$ times.

- Nested loops: using 4 nested loops which make the statement in the most inner loop to be repeated $10^{9}$ times in total.

The choice of these algorithms was based on the benchmarks used in the HOPE project [2].

We didn't use benchmarks that require array broadcasting since the implementation of array broadcasting in Python depends on numpy [30], which was not ported to Duby. Additionally, $\pi / 2$ calculation benchmark was replaced by nested loops benchmark in which we test the performance of behaviour of 4 nested loops: the reason for the change is that we wanted to increase the number of nested loops from 2 to 4 .

All benchmarks were executed on MacBook Air with 1.6 GHz Dual-Core Intel Core i5 CPU, 4 GB 1600 MHz Memory, running macOs Catalania version 10.15.7, with clang 1200.0.32.27, and Python 3.7.4. Python scripts were executed with -OO flag and Duby programs were compiled with -O3 flag.

The results are shown in Figure 4.

\section{UsING DUby FOR EdUCATION}

As computers are becoming a necessity in working and education environments, many students are already familiar with the concepts of files and filesystems when they start to learn programming.

Therefore, it would be natural to base programming education on these foundations. The memory in an introductory programming course could be treated as a filesystem and the memory objects as files.
With Duby's filesystem embedding students could manually build the memory hierarchy using popular file manipulation tools and then access and use it from within a program.

Consider the following "Hello, World" example:

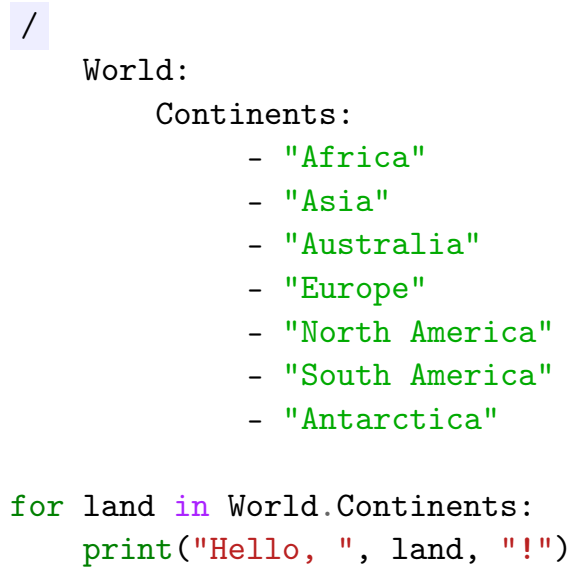

The example above uses filesystem-like memory hierarchy, introduces sequential and mapping data types, demonstrates iteration and, finally, prints the greeting messages to the output.

\section{ReLATEd Work}

\section{Motivation}

There are two seemingly contradictory tendencies in software engineering. One is to separate software functionality from state management and the other is to bind functionality and state management together.

The first goal lead to the adaptation of software design patterns, such as the model-viewcontroller [26], while the second goal lead object oriented languages to bind the functions and the data together.

Moreover, some object oriented languages have special language constructs, which allow the programmers to hide the data [10].

However, these two goals do not always contradict each other: there are examples of object oriented software designs that were tested by 


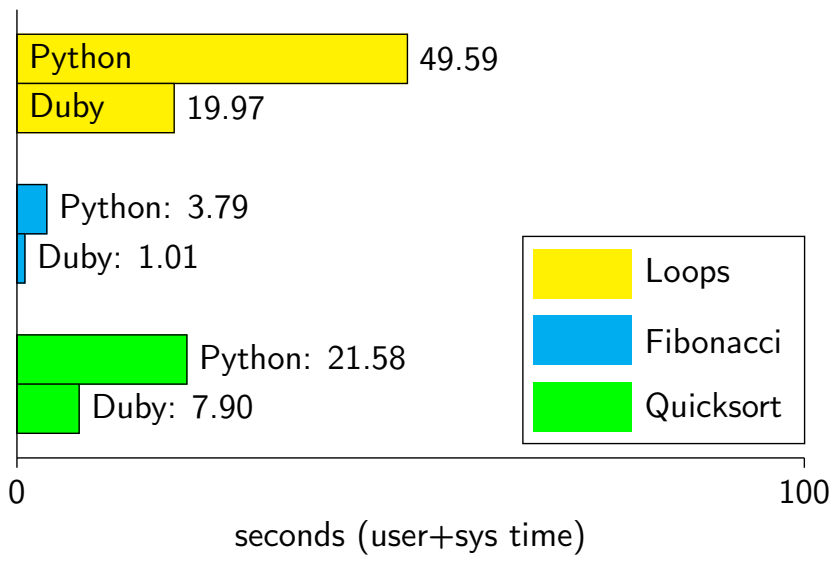

Figure 4: Benchmarks

time, in which the data was intentionally revealed.

Duby was inspired by the history of Document Object Model, abbreviated as DOM [31] and its influence on application development.

DOM was presented in the mid-1990's by Netscape Navigator and Internet Explorer (popular web browsers at that time) and has changed web development since then.

DOM was introduced as a programming interface which allowed to manipulate the documents displayed to the user by web browsers. Along with the JavaScript language, it was part of a machinery for making documents interactive: the document content could be altered in reaction to the user's actions.

As the browsers evolved, the documents presented to the users became more and more interactive and eventually the gap between a document and an application was blurred. When documents essentially became applications, the interface that allowed programs to manipulate the documents (DOM) became an interface for managing application states.

Today, DOM based UIs are widely used in web, mobile and desktop applications development [9].

One difference between DOM and statemachine programming is that state information in the former is stored in document elements, which can be identified by unique path strings, while state information structures in the latter are usually identified by an integer address.

Addressing memory items by integer numbers dates back to early programming languages and is related to the way hardware architectures are implemented.

Before the introduction of virtual memory, the numbers representing memory addresses were the indices of physical memory cells. However, since the introduction of virtual memory, the numbers representing memory addresses refer, in fact, to virtual memory cells [14].

Virtual memory abstraction allowed programming languages and their runtime systems to treat the virtual memory addresses as if they were physical.

Back in the early 1990's, when many of the popular programming languages used today, such as C++, Python or Java, already existed or were soon to be introduced, the typical amount of RAM in a personal computer ranged from a few megabytes to tens of megabytes and the typical hard disk capacity ranged from tens to hundreds of megabytes [20, 3]. These numbers significantly increased in the last few decades.

All of the standard libraries in the languages above allowed data files to be accessed using human-readable filesystem path strings [23, 11. 15, 24].

However, although the amount of RAM became larger, similar capabilities were for accessing values stored in memory were not added 
to the standard libraries of these languages.

In high-level programming languages like Java or Python, memory cell numbers could implicitly be bound to a reference variable [15, 24], while in low-level languages like $\mathrm{C}$ or $\mathrm{C}++$, those numbers were stored in pointer variables [18, 11].

Many programming languages store runtime type descriptors and offer access to variable types through reflection [13]. However, even in languages with introspective capabilities, programmers' access to memory addressing management is restricted.

\section{Education}

Programming languages education also evolved as hardware capabilities influenced the design of programming languages.

Introductory programming courses in the late 1970's used to begin with demonstration of code, which implements stateless algorithms that receive input and produce output. For such programs the memory could be treated as a big array of bytes that can be used during the execution of an algorithm [4].

However, as many programs do depend on state, focusing on stateless algorithms might not always be practical. The same code could execute a different algorithm for each different state configuration and as the number of algorithms described by a single piece of code grows, it becomes harder and harder to understand what the program does.

Therefore, during the 1990's some institutions began using object oriented programming approach in their introductory courses [27].

In object oriented programming the state of a program is separated into individual states of objects, which allows the number of different states that might affect a single algorithmic piece of code to be reduced. In this approach, the quality of a program depends on the quality of abstractions that lead to a clear separation of the global state. Therefore, in this method, great emphasis is put on teaching the particular way to enforce these abstractions (i.e. code encapsulation, etc.).
It is not easy to teach how to build a good object oriented abstractions, since if the abstractions are too coarse and generic then every such abstraction might still have to deal with many different states and that would miss the purpose of abstraction creation in the first place.

On the other hand, if the abstractions are more specific, then the number of such abstractions might be large, and therefore one might still have to understand the interactions between different abstractions to capture the program logic.

In the latter case, when the the memory is treated as an unstructured pool of objects it is not always easy to see how different objects affect each other.

Therefore, using a language which enforces the programmer to describe the structural relationships between objects in memory might be a good educational choice.

\section{Serialization}

Converting objects, which are stored in memory, memory to human-readable strings and vice versa is required in many areas such as communication, user interfaces, and datadriven applications.

Many programming languages provide builtin serialization mechanisms (serialization in Java, pickle [12] in Python).

The serialized data can be used for communication, since strings can be read by two independent sides, even if they are running in different environments and implemented in different languages.

Some languages are specifically targeted for communication. A widely used data interchange format is JSON [8].

\section{Memory-Based Storage}

The purpose of data interchange formats is, as their name suggests, to interchange data, rather than to store values in memory. DOM, which was discussed earlier, is being used for the latter purpose. 
DOM is a tree of elements, where every element can have a set of property pairs and a list of child elements. Manipulation of elements's tree changes the layout of the document.

Another way to store data in memory is to use in-memory databases, such as Redis and Memcached [34]. There are implementations of in-memory SQL databases as well. One of them is in-memory SQLite [21].

The concept of human-readable object hierarchy is similar to in-memory file system, where filesystem-like management techniques are also used for memory management.

Modern operating systems allow the definition of virtual filesystems in user space: FUSE, ProjFS, and PUFFS frameworks allow virtual filesystems to be created in Linux, Microsoft Windows and NetBSD, respectively. These frameworks can be used to create user-space in-memory filesystems [29].

\section{Static Typing in Dynamic Languages}

Static typing in often used in dynamic languages to optimize performance. For instance, a scripting language proposed in [32] integrates typed and untyped code.

The Cython language [5] enhances Python with explicit type declarations and is compiled directly to C. A just-in-time Numba [19] Python compiler allows to add static types to Python functions using Python's decorators syntax.

\section{Reference Counting}

Reference counting is a technique for garbage collection [7]. There are two disadvantages of using reference counting: one is the need for synchronization of the counters and the other is the possible creation of reference cycles. Both problems have been intensively studied, however, the latter problem is not completely solved: some programming languages provide weak references (i.e. references that do not influence the reference count) which allow the programmers to tackle this problem manually.

\section{Conclusion and Future DIRECTIONS}

The initial goal of dual binding mechanism was to improve program comprehension by substituting integer addresses by human-readable unique path strings. However, we see that dual binding can be used to benefit memory management and performance optimization as well.

The underlisted optimization directions can be further investigated.

Duby allows a restricted form of static typing, and since Python allows type annotations, optimized code can be generated for functions which receive such types as arguments. Moreover, type hints received from a dynamic analysis could be used to decide which optimized functions should be created. Functions receiving automatic variables could perform type verification and call the optimized function in case the actual types match the hints.

Another possible optimization is copy-onwrite implementation. Since Duby uses deepcopying of values for every assignment of an automatic variable, making multiple assignments of the same values has a performance cost. Some of this copying might be spared if the values are not changed after the assignment. A copy-on-write instrumentation could be written for automatic variables to reduce the number of performed deep-copies. Moreover, dynamic analysis could register values which are changed after the assignment, and the compiler could use this hint to decide whether to use copy-on-write semantic or not for every variable.

Since automatic can be placed on stack and not allocated on the heap, dynamic analysis could be used to predict for every function the potential required stack size for its automatic variables. Then all automatic variables would be put by default on stack, and only in exceptional cases (when the prediction of the required amount of memory turns out to be wrong) would values be allocated on the heap.

Another possible research direction is the implementation of different memory models 
which utilize the memory tree. A separate memory tree branch could be automatically dedicated to a thread and this could suggest new ways of building inter-thread communication.

\section{REFERENCES}

[1] Alfred V Aho, Ravi Sethi, and Jeffrey D Ullman. Compilers, principles, techniques. Addison wesley, 7(8):9, 1986.

[2] J. Akeret, L. Gamper, A. Amara, and A. Refregier. Hope: A python just-in-time compiler for astrophysical computations. Astronomy and Computing, 10:1 - 8, 2015. ISSN 2213-1337. doi: https://doi.org/ 10.1016/j.ascom.2014.12.001. URL http: //www.sciencedirect.com/science/ article/pii/S2213133714000687

[3] Centris 650: Technical Specifications. Apple Inc. URL https://support.apple.com/ $\mathrm{kb} / \mathrm{SP} 230$

[4] Richard H Austing, Bruce H Barnes, Della T Bonnette, Gerald L Engel, and Gordon Stokes. Curriculum'78: recommendations for the undergraduate program in computer science-a report of the $\mathrm{acm}$ curriculum committee on computer science. Communications of the ACM, 22(3): 147-166, 1979.

[5] Stefan Behnel, Robert Bradshaw, Craig Citro, Lisandro Dalcin, Dag Sverre Seljebotn, and Kurt Smith. Cython: The best of both worlds. Computing in Science $\mathcal{E}$ Engineering, 13(2):31-39, 2011.

[6] Oren Ben-Kiki, Clark Evans, and Brian Ingerson. Yaml ain't markup language (yaml $^{\mathrm{TM}}$ ) version 1.1. Working Draft 200805, 11, 2009.

[7] Stephen M Blackburn and Kathryn S McKinley. Ulterior reference counting: Fast garbage collection without a long wait. In Proceedings of the 18th annual ACM
SIGPLAN conference on Object-oriented programing, systems, languages, and applications, pages 344-358, 2003.

[8] Pierre Bourhis, Juan L Reutter, Fernando Suárez, and Domagoj Vrgoč. Json: data model, query languages and schema specification. In Proceedings of the 36th ACM SIGMOD-SIGACT-SIGAI symposium on principles of database systems, pages 123135, 2017.

[9] Hugo Brito, Anabela Gomes, Álvaro Santos, and Jorge Bernardino. Javascript in mobile applications: React native vs ionic vs nativescript vs native development. In 2018 13th Iberian Conference on Information Systems and Technologies (CISTI), pages 1-6. IEEE, 2018.

[10] Bruce Eckel. Thinking in C++. Prentice Hall, 1999.

[11] Margaret A Ellis and Bjarne Stroustrup. The annotated $\mathrm{C}++$ reference manual. Addison-Wesley, 1990.

[12] Laurent Fasnacht. mmappickle: Python 3 module to store memory-mapped numpy array in pickle format. Journal of Open Source Software, 3(26):651, 2018.

[13] Ira $\mathrm{R}$ Forman, Nate Forman, and John Vlissides Ibm. Java reflection in action. 2004.

[14] Robert A Gingell, Joseph P Moran, and William A Shannon. Virtual memory architecture in sunos. AUUGN, 8(5):128, 1987.

[15] James Gosling and Henry McGilton. The java language environment. Sun Microsystems Computer Company, 2550:38, 1995.

[16] Dan Ingalls, Ted Kaehler, John Maloney, Scott Wallace, and Alan Kay. Back to the future: the story of squeak, a practical smalltalk written in itself. In Proceedings of the 12th ACM SIGPLAN conference on Object-oriented programming, systems, languages, and applications, pages 318-326, 1997. 
[17] Nicolai M Josuttis. The C++ standard library: a tutorial and reference. AddisonWesley, 2012.

[18] Brian W Kernighan and Dennis M Ritchie. The C programming language. 2006.

[19] Siu Kwan Lam, Antoine Pitrou, and Stanley Seibert. Numba: A llvm-based python jit compiler. In Proceedings of the Second Workshop on the LLVM Compiler Infrastructure in HPC, pages 1-6, 2015.

[20] Peter H. Lewis. Personal computers; i.b.m. puts more power in ps/1's. New York Times, page 13-13, Oct 1993. URL https://www. nytimes.com/1993/10/12/science/ personal-computers-ibm-puts-more-pow html.

[21] Mike Owens and Grant Allen. SQLite. Springer, 2010.

[22] Nikolaos S Papaspyrou. A formal semantics for the c programming language. Doctoral Disseration. National Technical University of Athens. Athens (Greece), 15, 1998.

[23] Dennis M Ritchie. The c programming language-reference manual. ATET Bell Laboratories, 1980.

[24] Guido Rossum. Python reference manual. 1995.

[25] Manuel Serrano and Pierre Weis. Bigloo: a portable and optimizing compiler for strict functional languages. In International Static Analysis Symposium, pages 366-381. Springer, 1995.

[26] Kishori Sharan. Model-view-controller pattern. In Learn JavaFX 8, pages 419-434. Springer, 2015.

[27] Suzanne Skublics and Paul White. Teaching smalltalk as a first programming language. In Proceedings of the twenty-second SIGCSE technical symposium on Computer science education, pages 231-234, 1991.
[28] Guido Van Rossum and Fred L Drake. The python language reference manual. Network Theory Ltd., 2011.

[29] Bharath Kumar Reddy Vangoor, Vasily Tarasov, and Erez Zadok. To \{FUSE\} or not to $\{$ FUSE $\}$ : Performance of user-space file systems. In 15th $\{$ USENIX\} Conference on File and Storage Technologies ( $\{F A S T\} 17)$, pages 59-72, 2017.

[30] Stéfan van der Walt, S Chris Colbert, and Gael Varoquaux. The numpy array: a structure for efficient numerical computation. Computing in science $\mathcal{E}$ engineering, 13 (2):22-30, 2011.

[31] Lauren Wood, Arnaud Le Hors, Vidur Apparao,SSteve Byrne, Mike Champion, Scott Isaacs, İan Jacobs, Gavin Nicol, Jonathan Robie, Robert Sutor, et al. Document object model (dom) level 1 specification. W3C recommendation, 1, 1998.

[32] Tobias Wrigstad, Francesco Zappa Nardelli, Sylvain Lebresne, Johan Östlund, and Jan Vitek. Integrating typed and untyped code in a scripting language. In Proceedings of the 37th annual ACM SIGPLAN-SIGACT symposium on Principles of programming languages, pages 377-388, 2010.

[33] Alon Zakai. Emscripten: an llvm-tojavascript compiler. In Proceedings of the ACM international conference companion on Object oriented programming systems languages and applications companion, pages 301-312, 2011.

[34] Hao Zhang, Bogdan Marius Tudor, Gang Chen, and Beng Chin Ooi. Efficient inmemory data management: An analysis. Proceedings of the VLDB Endowment, 7(10): 833-836, 2014. 